\title{
Pleurisy Can Cause Chest Wall Tenderness: A Case Report
}

\author{
Shaul Yaari, Elchanan Juravel, Murad Daana, Samuel N Heyman \\ Department of Medicine, Hadassah Hebrew University Hospital, Mt. Scopus, Jerusalem, Israel
}

Received: $12 / 04 / 2020$

Accepted: $18 / 06 / 2020$

Published: $23 / 07 / 2020$

How to cite this article: Yaari S, Juravel E, Daana M, Heyman SN. Pleurisy can cause chest wall tenderness: a case report. EJCRIM 2020;7: doi:10.12890/2020_001657.

Conflicts of Interests: The Authors declare that there are no competing interests.

This article is licensed under a Commons Attribution Non-Commercial 4.0 License

\section{ABSTRACT}

Stab-like localized chest pain, aggravated by breathing, is compatible with pleuritic pain or with aching related to chest wall abnormalities. Local tenderness inflicted by palpation helps to differentiate pleuritic from musculoskeletal chest pain and serves as a principal accessory manoeuvre in the algorithm of chest pain evaluation.

Herein, we report the case of a 27-year-old patient with pulmonary thromboembolism and right lower lobe consolidation/atelectasis. The patient presented with right-sided chest pain, radiating to the shoulder, related to pleural irritation, yet associated with confounding intense chest wall tenderness and guarding, also involving the costovertebral angle. We propose that spinal reflex-related chest wall tenderness was involved, similar to peritoneal signs evoked by irritation of the parietal peritoneum.

This case report illustrates that localized chest wall tenderness and guarding, triggered by palpation, may not serve as unequivocal indicators of musculoskeletal pain, and could be unrecognized features of pleuritic chest pain also.

\section{LEARNING POINTS}

- Pleuritic chest pain may be associated with local tenderness and guarding.

- These unrecognized features of pleurisy supposedly reflect a spinal reflex, initiated by nociceptors in the parietal pleura.

- Local pain inflicted upon palpation and guarding may not serve as unequivocal indicators of musculoskeletal chest pain.

\section{KEYWORDS}

Pulmonary embolism, chest pain, pleura, symptoms, physical examination

\section{INTRODUCTION}

Chest pain is one of the most common complaints, challenging physicians in primary care settings and in departments of emergency medicine. The clinical assessment of chest pain is initiated by defining the nature of the pain, principally using medical history and physical examination. Stab-like localized pain, aggravated by breathing, is compatible with pleuritic pain or with aching related to chest wall abnormalities. Local tenderness inflicted by palpation helps to differentiate pleuritic from musculoskeletal chest pain and serves as a principal accessory manoeuvre in the algorithm of chest pain evaluation ${ }^{[1]}$. Although features of both pleuritic and musculoskeletal chest pain patterns help exclude myocardial ischaemia by rapid rule-out protocols ${ }^{[2]}$, distinguishing between these 2 pain entities is diagnostically essential and quite simple, as most causes of pleurisy, including pulmonary thromboembolism, are not associated with chest wall soreness ${ }^{[3]}$. Herein, we report the case of a patient with pulmonary thromboembolism and chest pain related to pleural irritation, yet associated with confounding intense chest wall tenderness and guarding, also involving the costovertebral angle, likely reflecting pain mechanisms similar to those evoked by irritation of the parietal peritoneum. 


\section{CASE PRESENTATION}

A 27-year-old female patient using a contraceptive vaginal ring (NuvaRing ${ }^{\circledR}$ ) presented with progressive severe right-sided chest pain for 2 days, radiating to the shoulder and scapula, aggravated by breathing and the supine position, and ameliorated by bending forward. Physical examination disclosed splinting of the right hemithorax, and dullness on percussion and reduced breath sounds at the right lung base, with diminished right lung expansion and minimal downward movement of the right hemidiaphragm upon inspiration. Intense muscle tenderness was noted along the posterior and lateral rim of the ribcage and at the costovertebral angle. The remaining physical examination was unremarkable, and signs of peritoneal or retroperitoneal irritation were absent.

Radiocontrast-enhanced computerized tomography, illustrated in Fig. 1, revealed multiple pulmonary emboli and right lower lobe consolidation/ atelectasis, compatible with infarcts involving basal segments adjacent to the diaphragm and lower chest wall, with hypoaeration of the right lung and a small pleural effusion.

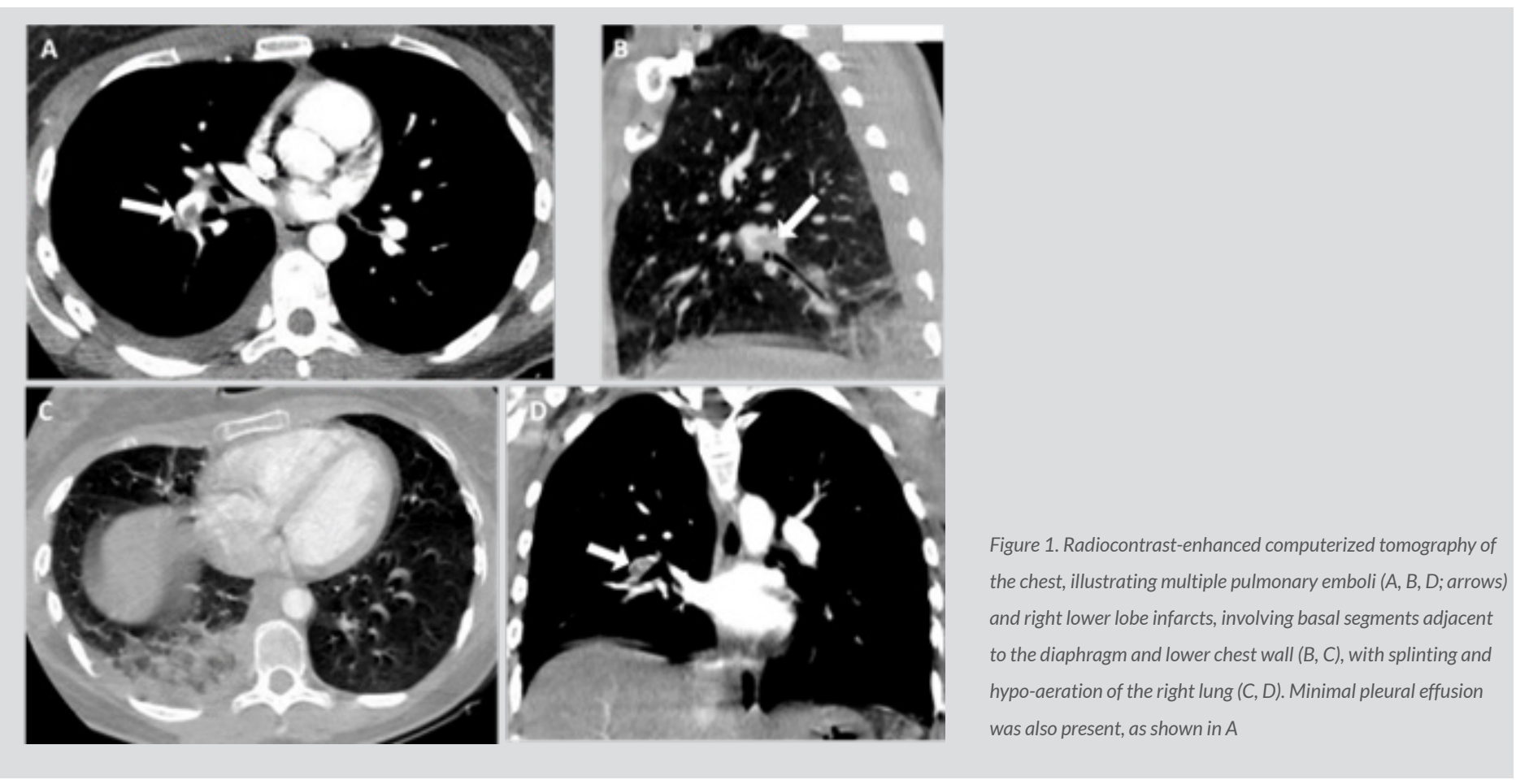

Other differential diagnoses, including subdiaphragmatic and retroperitoneal disorders, were excluded on a clinical basis and by additional laboratory and imaging modalities.

The patient was treated with fractionated heparin and subsequently with apixaban, with a prompt resolution of her symptoms within 48 hours. The contraceptive vaginal ring has been removed and further workup for thrombophilia is currently underway.

\section{DISCUSSION}

Inflamed pleura produces pleuritic chest pain, originating from nociceptors in the parietal pleura and delivered via afferent fibres in deep intercostal nerves and along the phrenic nerve ${ }^{[1]}$. This patient with extensive thromboembolic infarction of the right lower lobe presented with typical symptoms of pleuritic chest pain. The ache radiating to the shoulder specifically characterizes mid-diaphragmatic irritation, with sensorial input conducted along the phrenic nerve ${ }^{[1]}$. The physical findings and imaging features indicate a well-known reflective reduction of aeration of the right lung, that somewhat alleviates the suffering ${ }^{[4]}$.

However, the prominent muscle tenderness in our patient, which involved mainly the lower posterior and lateral chest wall and costovertebral angle, is not an acknowledged component of pleuritic pain and suggests musculoskeletal origin or alternative diagnoses, including subdiaphragmatic and retroperitoneal disorders. Nevertheless, we propose that the muscle tenderness and chest wall guarding, reported here in the context of evident pleuritic irritation, may be unrecognized features of pleurisy, supposedly reflecting a spinal reflex, initiated by nociceptors in the parietal pleura ${ }^{[5]}$, resembling abdominal guarding caused by inflamed parietal peritoneum. 


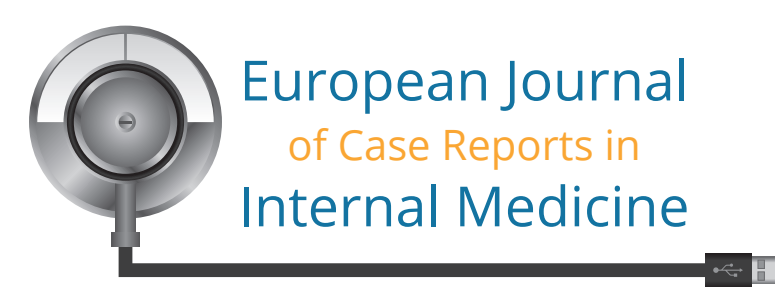

Indeed, local tenderness and muscle rigidity, associated with guarding and rebound are comparable familiar features of peritoneal irritation. They represent a spinal reflex, initiated by nociceptors in the parietal peritoneum, with an efferent arm consisting of motor neurons in the anterior horn of the spinal cord, innervating abdominal wall muscles. Such a phenomenon involving the chest wall likely also explains some characteristics of Coxsackie B-related pleurodynia (Bornholm disease), that have traditionally been attributed to costochondritis and myositis.

In conclusion, we suggest that localized chest wall pain triggered by palpation with muscle ache and guarding may not serve as unequivocal indicators of musculoskeletal pain, and could be unrecognized features of pleuritic pain also.

\section{REFERENCES}

1. Brims FJ, Davies HE, Lee YC. Respiratory chest pain: diagnosis and treatment. Med Clin North Am 2010;94(2):217-232

2. Huis In 't Veld MA, Cullen L, Mahler SA, Backus BE, Dezman ZDW, Mattu A. The fast and the furious: low-risk chest pain and the rapid rule-out protocol. West J Emerg Med 2017;18(3):474-478.

3. Kline JA, Kabrhel C. Emergency evaluation for pulmonary embolism, part 1: clinical factors that increase risk. J Emerg Med 2015;48(6):771-780.

4. Reamy BV, Williams PM, Odom MR. Pleuritic chest pain: sorting through the differential diagnosis. Am Fam Physician 2017;96(5):306-312.

5. Smolin LN, Frankstein SI. Reciprocal segmental muscle reflexes at the thoracic level of the spinal cord. Exp Neurol 1979;63(2):443-446. 